\title{
XMCCTree: an algorithm for producing Mcctree in incompact structure
}

\begin{abstract}
Different notions and techniques have been proposed to support query in XML. This led to the generation of algorithms to provide more efficient and accurate query processing. This research proposes an efficient algorithm to support keyword query in XML document. The propose algorithm, namely XMCCTree, employs MCLCA notion with some modifications in order to provide more efficient algorithm.
\end{abstract}

Keyword: Efficient algorithm; Keyword query; XMCCTree; XML query; XML structure 